\title{
Spherical Tokamak Development in Brazil
}

\author{
G.O. Ludwig, E. Del Bosco, J.G. Ferreira, L.A. Berni, R.M. Oliveira, \\ M.C.R. Andrade, C.S. Shibata, M. Ueda, L.F.W. Barbosa*, \\ and the high-power microwave sources group, \\ J.J. Barroso, P.J. Castro, and H. Patire Jr ${ }^{\dagger}$ \\ Laboratório Associado de Plasma, LAP \\ Instituto Nacional de Pesquisas Espaciais, INPE \\ 12227-010, São José dos Campos, SP, Brazil
}

Received on 18 March, 2003. Revised version received on 19 August, 2003.

\begin{abstract}
This paper describes the general characteristics of spherical tokamaks, or spherical tori, with a brief overview of work in this area already performed or in progress at several institutions worldwide. The paper presents also the steps in the development of the ETE (Experimento Tokamak Esférico) project, its research program, technical characteristics and operating conditions as of December, 2002 at the Associated Plasma Laboratory (LAP) of the National Space Research Institute (INPE) in Brazil.
\end{abstract}

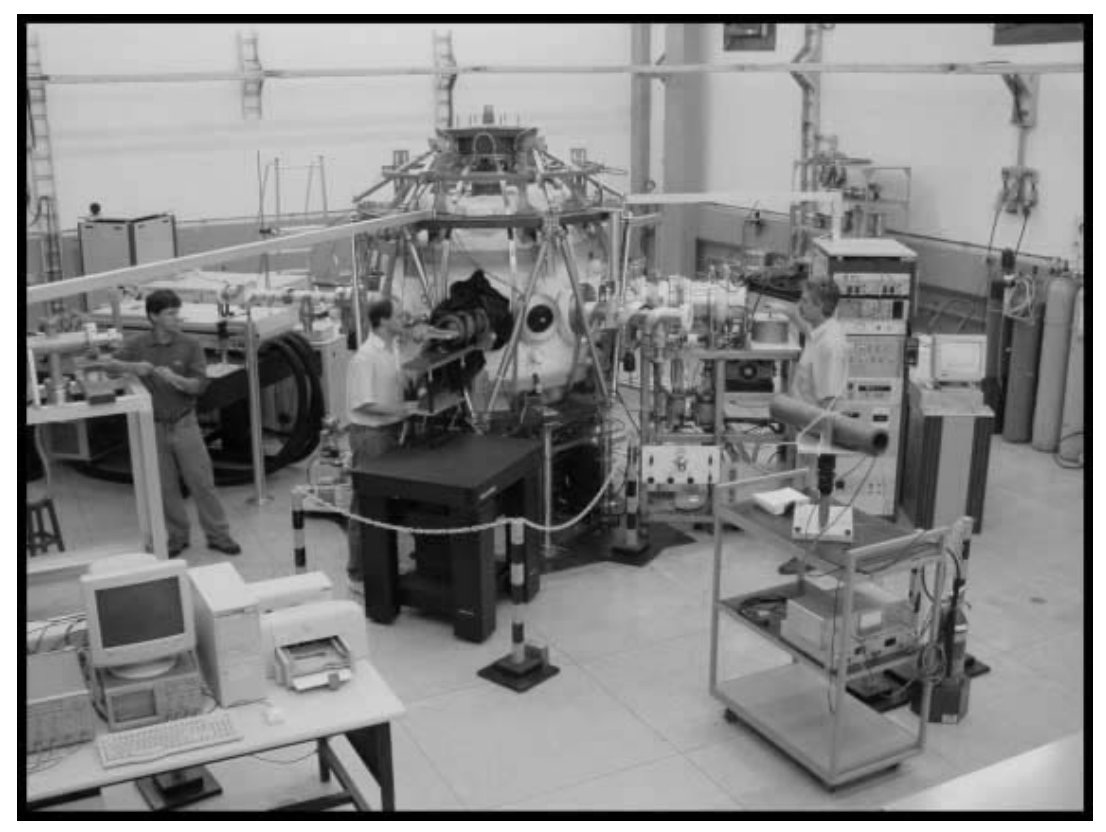

Figure 1. An overall view of the ETE spherical tokamak experiment.

\section{Introduction}

The ETE spherical tokamak (Experimento Tokamak Esférico) started its operational phase in late 2000 at the As- sociated Plasma Laboratory of the National Space Research Institute in Brazil (ref Figure 1). It is devoted to the study of the operating regimes, confinement properties and current drive schemes in a low aspect ratio tokamak plasma configu-

\footnotetext{
*Present Address: Faculdade de Engenharia, Arquitetura e Urbanismo, FEAU, Universidade do Vale do Paraíba, UNIVAP, 12244-000, São José dos Campos, SP, Brazil

${ }^{\dagger}$ Present Address: Divisão de Mecânica Espacial e Controle, DMC, Coordenação Geral de Engenharia e Tecnologia Espacial, ETE, Instituto Nacional de Pesquisas Espaciais - INPE
} 
ration. This hot plasma magnetic confinement configuration [1] has been a subject of experimental study for a little over than one decade, offering the prospect of compact volumetric neutron sources and low-cost fusion power plants. Besides exploring the properties of low aspect ratio tokamaks, the ETE experiment will allow diagnostics development and training in tokamak operation, with a research program open to collaboration with other institutions. The initial operation of ETE is limited to the study of inductively heated plasmas. In the future, with the implementation of auxiliary heating methods, it will be possible to explore improved confinement regimes and means of current drive for steady-state operation. The large number of physics problems and technical issues that remain to be explored and solved in the spherical torus configuration allow to perform, even in a modest device, a research program that can contribute effectively to fusion research.

\section{Spherical tokamak experiments worldwide}

The spherical tokamak concept was tested in the late eighties by the insertion of current-carrying central rods into small existing devices [2][3][4]. These experiments were limited to cold plasmas (electron temperatures of a few tenths of eV) but gave evidence to the advantage of combining the favorable characteristics of both the compact torus and the conventional tokamak. Figure 2 shows the location of the spherical torus (ST) in the three dimensional $(A, q, \beta)$ space, relative to the spheromak, field-reversed configuration (FRC), conventional tokamak, and reversed-field pinch (RFP) devices. The aspect ratio $A=R_{0} / a$ is the ratio of the major to the minor radius of the plasma torus, the safety factor $q$ is related to the rate of change of toroidal flux with poloidal flux, and the $\beta$ value gives the ratio of the plasma kinetic pressure to the magnetic pressure. The elongation factor $\kappa$, which gives the ratio of the length of the poloidal cross section along the symmetry axis to the length along the equatorial plane, can be seen on this diagram from the plasma outline drawings.

The START (Small Tight Aspect Ratio Tokamak) experiment operated from 1991 to 1998 and was the world's first tokamak to study hot plasmas $(>100 \mathrm{eV})$ at aspect ratios $A<2$ [5][6]. A full account of START results is presented in reference [7]. It was the first representative of a series of worldwide spherical tokamak experiments put into operation in the nineties, with main parameters listed in Table 1. In some cases, the parameters given in this table correspond to nominal values that may not have been reached yet. In the remaining of this section, a succinct description of the methods of plasma formation used in these devices is presented, showing the similarities between the research programs carried out by the ST and compact torus communities.

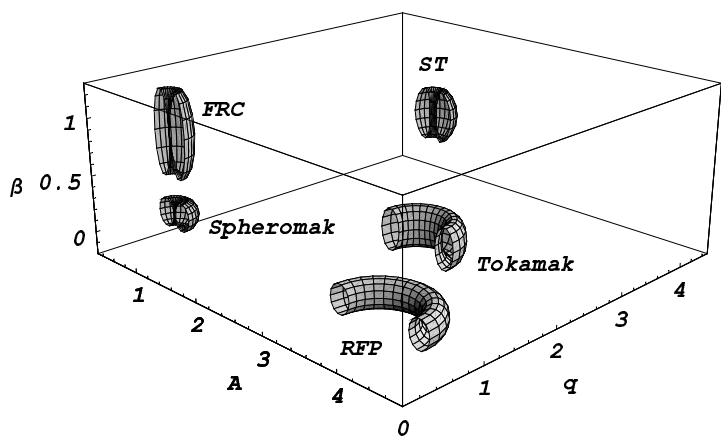

Figure 2 Axisymmetric configurations. Spherical tokamaks present both the strong toroidicity effects of compact tori (notably magnetic shear) and the good stability properties provided by the external toroidal field of conventional tokamaks.

Table 1. Spherical tokamaks worldwide: (1) GB (shutdown), (2) GB, (3) US, (4) RU, (5) JP, (6) BR, (7) CN (commissioning), (8) IT (proposed).

\begin{tabular}{|c|c|c|c|c|c|c|}
\hline Device & $\begin{array}{c}R_{0} \\
\mathbf{m}\end{array}$ & $\begin{array}{c}a \\
\mathbf{m}\end{array}$ & $\kappa$ & $\begin{array}{c}B_{0} \\
\mathbf{T}\end{array}$ & $\begin{array}{c}I_{p} \\
\mathbf{M A}\end{array}$ & $\begin{array}{c}P_{\text {aux }} \\
\mathbf{M W}\end{array}$ \\
\hline LATE $^{5}$ & 0.2 & 0.14 & 1.2 & 0.18 & 0.05 & 0.35 \\
TS-3 $^{5}$ & 0.2 & 0.14 & 2 & 0.2 & 0.08 & - \\
Proto-Sphera $^{8}$ & 0.2 & 0.16 & 2.2 & 0.06 & 0.18 & - \\
HIT-II $^{3}$ & 0.3 & 0.2 & 1.8 & 0.5 & 0.25 & - \\
ETE $^{6}$ & 0.3 & 0.2 & 1.8 & 0.6 & 0.4 & - \\
SUNIST $^{7}$ & 0.3 & 0.23 & 1.8 & 0.15 & 0.05 & - \\
HIST $^{5}$ & 0.3 & 0.24 & 2 & 0.2 & 0.1 & - \\
START $^{1}$ & 0.32 & 0.25 & 3 & 0.31 & 0.31 & 1 \\
CDX-U $^{3}$ & 0.34 & 0.22 & 1.6 & 0.23 & 0.07 & - \\
TST- $^{5}$ & 0.36 & 0.23 & 1.8 & 0.4 & 0.2 & - \\
Globus-M $^{4}$ & 0.36 & 0.24 & 2.2 & 0.62 & 0.5 & 3.7 \\
Pegasus $^{3}$ & 0.45 & 0.39 & 3 & 0.1 & 0.2 & 1 \\
TS-4 $^{5}$ & 0.5 & 0.42 & 3 & 0.5 & 0.3 & - \\
MAST $^{2}$ & 0.85 & 0.65 & 3 & 0.52 & 2 & 6.4 \\
NSTX $^{3}$ & 0.85 & 0.68 & 2.5 & 0.6 & 1.5 & 13 \\
\hline
\end{tabular}

Excepting LATE, the devices listed in Table 1 use inductive methods for plasma formation or sustainment, either transferring flux from an internal solenoid or trapping an initial bias flux through the rapid reversal or increase of the current in external poloidal coils. The external coils method is less efficient than the standard ohmic heating technique due to limitations in the retention of the initial bias flux. Thus far, the sustainment of the spherical torus configuration, which depends in large part of the internal plasma currents, awaits the development of efficient non-inductive current drive techniques. This is an absolute requirement for steady-state operation of future reactors.

The previous paragraph implies the objective of the LATE (Low Aspect ratio Torus Experiment) device, which is to achieve start-up and formation of spherical tokamak plasmas by electron cyclotron heating and current drive (ECH/ECCD) only, without using inductive methods [8]. This was also the initial objective of the CDX-U (Current Drive Experiment-Upgrade) device, which has been used recently in experiments involving the use of liquid lithium as a 
plasma facing component [9]. In the search for current drive amplification methods, the TS-3 (Tokyo University Spherical Torus-3) device was used, during its initial operating phase, to conduct tokamak-merging experiments and investigate the relaxation and reconnecting mechanisms. In TS-3 the toroidal plasma is typically formed starting from the arc current between the external electrodes of two plasma guns, i.e., using DC helicity injection start-up, plus magnetic compression. Presently, the high power heating of the magnetic reconnection is being used in TS-3 and the larger TS- 4 device to study the high- $\beta$ stability of spherical tori. In these experiments two spheromaks with opposite toroidal fields are merged to form an oblate FRC that is subsequently transformed into an spherical tokamak configuration by applying the external toroidal field [10].

The principal objective of the Proto-SPHERA experiment is to test an ultra low aspect ratio tokamak, with the central conductor replaced by an arc discharge, as a prototype for a larger SPHERA (Spherical Plasma for HElicity Relaxation Assessment) device [11]. In this concept an initial screw-pinch is driven unstable by increasing the arc current and then relaxes to a stable flux core spheromak state, similarly to the TS-3 experiment. Helicity injection and dissipation inside a torus is the central research topic of the HIT-II (Helicity Injected Tokamak-II) device, which uses both transformer action and coaxial helicity injection (CHI) to drive toroidal current in the spherical tokamak geometry [12]. According to the experimental results of HIT-II, the $n=1$ external kink mode drives toroidal current in the plasma core by dynamo action, relaxing the $\mathrm{CHI}-$ driven hollow current profile. The roles of helicity conservation and $n=1$ relaxation activity in spherical tokamak plasmas during the helicity injection phase are also the subject of study of the HIST (Helicity Injected Spherical Torus) experiment [13].

The Pegasus toroidal experiment is an extremely low aspect ratio device with the goal of minimizing the central column while maintaining good confinement and stability [14]. Using up to $1 \mathrm{MW}$ of radio frequency (RF) power this experiment aims to demonstrate plasma heating and current drive with high-harmonic fast waves at $A$ near unity. RF plasma heating and stability are also topics of research in the TST-2 (Tokyo Spherical Tokamak-2) experiment, where the excitation and propagation of high-harmonic fast waves, emission of electron Bernstein waves, and physics of internal reconnection events [15] have been recently studied. SUNIST (Sino United Spherical Tokamak) is a new facility undergoing tests that will combine, in a second phase, electron cyclotron resonance start-up with fast wave current drive and, if successful, should permit non-inductive operation [16].

In 1999 a series of spherical tokamak experiments started operating with significant plasma current and auxiliary power capability. The Globus-M (Globus-Modified) project is dedicated to basic spherical tokamak physics research up to the $0.5 \mathrm{MA}$ plasma current level, with emphasis in auxiliary heating and current drive using various radio frequency methods: ion cyclotron heating (1-1.5 MW, 10$15 \mathrm{MHz}$ ), high harmonic fast waves (0.7 MW, 30-50 MHz), lower hybrid schemes $(<0.5 \mathrm{MW}, 2.45 \mathrm{GHz})$, and neutral beam heating $(\sim 1 \mathrm{MW}, 30 \mathrm{kV})$. During 2002 the plasma current in Globus-M reached 0.36 MA [17]. The objectives of MAST (Mega Ampere Spherical Tokamak) are to enhance the tokamak database and to explore the physics of the spherical torus (understand confinement scaling, magnetohydrodynamic stability, plasma exhaust, etc.) at plasma currents in the 1 2 MA level. The low aspect ratio configurations in MAST are obtained by a dynamic method using external induction coils and a vertical field to compress the plasma towards the central column, which is the same inductive start-up method employed previously in START. A report of the results in MAST until mid 2001 is presented in reference [18]. By the end of 2001, H-mode was attained in purely ohmic discharges in MAST [19]. Finally, the NSTX (National Spherical Torus Experiment) is dedicated to explore advanced spherical torus regimes in the $1 \mathrm{MA}$ range of plasma current. Very large power auxiliary heating and current drive systems are available: neutral beam injection $(7 \mathrm{MW}, 100 \mathrm{kV})$ and high harmonic fast waves $(6 \mathrm{MW}$, $30 \mathrm{MHz}$ ). One of the most interesting results is the current initiation with $\mathrm{CHI}$ up to the $0.4 \mathrm{MA}$ level. A full report of results in the NSTX device is presented in reference [20].

\section{Conceptual design of ETE}

The ETE spherical tokamak is a prototype of the Experimento Toroidal Avançado - ETA (Advanced Torus Experiment), the conceptual design of which was elaborated in 1986. This concept consisted of a spherical torus with aspect ratio $A \leq 1.5$, major radius $R_{0}=0.3 \mathrm{~m}$, elongation $\kappa \geq 1.6$, toroidal field $B_{0}=0.6 \mathrm{~T}$, and plasma current up to $I_{p}=0.8 \mathrm{MA}$ to explore means of radio frequency current drive. In the original proposal the plasma would be formed by electron cyclotron resonance heating and the current driven by lower-hybrid $(0.8 \mathrm{MW})$ and electron cyclotron $(0.4 \mathrm{MW})$ waves. For the low-density phase it was felt that the current could be ramped up with the experimentally achieved current drive efficiencies for lower-hybrid waves. In the high-density, warm plasma regime, the wave absorption would be governed by the absorption of electron Bernstein waves (EBW), generated by mode conversion of the incoming extraordinary waves at the upper hybrid resonance (UHR) [21]. For even higher temperatures, direct cyclotron damping at the electron cyclotron resonance (ECR) layer becomes important, and absorption of the incoming waves (injected from the high-field side) would occur before reaching the UHR layer. RF power in the electron cyclotron range would be provided by the $30 \mathrm{GHz}, 100 \mathrm{~kW}$ gyrotrons under development in Brazil at that time, with additional heating provided by a charge and current neutralized intense ion beam. It was expected that a volume averaged beta $10 \sim 20 \%$ could be reached in the experiment without exciting dangerous magnetohydrodynamic instabilities [22][23]. 


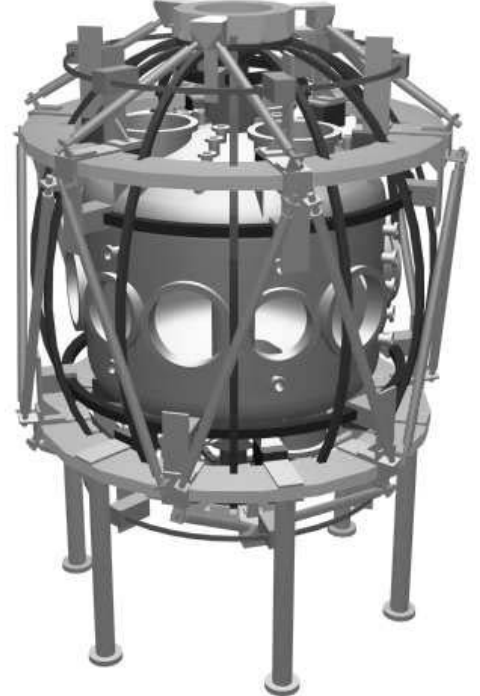

Figure 3. Artistic view of ETE.

It was soon realized the need to include inductive means of plasma current formation to avoid the uncertainties related to the RF methods, and a small solenoid was introduced in subsequent versions of the design [24][25][26]. Even at the present time, many of the RF schemes have not been properly tested in spherical tokamaks. In particular, the plasma heating and current drive method based on EBW remains one of the interesting possibilities in the future [18]. Lacking appropriate financial support for a large size machine, a minimal design for a spherical tokamak was completed in 1993 [27] leading to the present ETE device, which is illustrated in Figure 3. The detailed engineering design was then carried out, with manufacturing of some components beginning in mid 1995 [28][29][30]. Assembly began in mid 1998 after completion of the new experimental hall built at INPE [31]. Tests of the machine started in late 1999 and the first tokamak plasma was attained in 28 November 2000 [32].

\section{Research program of ETE}

The experimental program of ETE focuses on the study of the physics of spherical tokamak plasmas. The program started with plasmas in the ohmic regime, optimized by good vacuum conditioning and pre-ionization. Adequate control of the particle recycling conditions at the edge will be crucial for a low impurity discharge. An operation parameters space study will be carried out, looking for high-density limits and lowest ratio of the central column current to plasma current. The implementation of a fast neutral lithium beam probe and an array of electrostatic probes will allow a detailed study of the plasma edge conditions. This is an important issue, since it is presently accepted that the plasma boundary processes have a strong influence on the core plasma behavior. Magnetic activity will be monitored during the entire research program.
The plasma diagnostic system of ETE also includes the basic electromagnetic diagnostics, optical spectroscopy, optical imaging and Thomson scattering, already installed, and a far infrared laser interferometry system in the near future. Soft X-rays imaging in three planes will be attempted in order to estimate the position of the magnetic axis and the elongation at the axis, which should improve the results of the magnetic reconstruction procedure, still to be implemented.

By increasing the available capacitor banks the plasma current can be extended from the presently attainable $0.2 \mathrm{MA}$ range to more than $0.4 \mathrm{MA}$ with a $50 \mathrm{~ms}$ pulse duration (limited by solenoid stress and heating). The maximum allowable value of the magnetic field is $0.8 \mathrm{~T}$ (mechanical stress limit), depending also on an upgrade of the capacitor banks. Means of auxiliary heating and current drive, which will lead to studies of improved plasma confinement, have been just recently addressed, in collaboration with the University of São Paulo in the area of radio frequency plasma heating. Auxiliary heating and current drive is envisaged up to the $1 \mathrm{MW}$ level.

Theoretical work has been carried out during the last few years notably in the areas of self-consistent equilibrium calculations including neo-classical effects, plasma start-up models including eddy current effects, and - looking to applications of high-power microwave sources in ETE - propagation and interaction of intense electron beams with radio frequency fields in resonant cavities and corrugated waveguides. Modelling of the scrape-off layer and studies of the plasma edge physics will be undertaken in the near future.

In general terms, the objective of the ETE experiment is to explore the physics of plasmas in the spherical torus geometry. In particular, it is planned to investigate aspects of the plasma edge physics and the physics of plasma heating by radio frequency waves. Besides research in low aspect ratio tokamaks and diagnostics development, another important objective of the ETE program is training in tokamak operation. The ETE tokamak is open to collaboration with other laboratories and universities, both national and international, providing the only spherical tokamak facility available for training in the southern hemisphere.

\section{Technical characteristics of ETE}

The main parameters of ETE are listed in Table 2. During the initial operation a modestly high plasma current $I_{p} \cong$ $0.2 \mathrm{MA}(\sim 15 \mathrm{~ms})$ will be produced in a 1.5 aspect ratio configuration with a toroidal field $B_{0} \leq 0.4 \mathrm{~T}(\sim 100 \mathrm{~ms})$. These parameters should be attained with fully inductive current drive using the capacitor banks presently available for the ohmic and toroidal field circuits. However, the maximum parameters will be reached only after upgrading the capacitor banks. By increasing the stored energy one expects also to increase the pulse length to values that will permit a better study of the plasma confinement properties. The ultimate parameter values are limited by mechanical stresses in the demountable joints of the toroidal field coil $\left(B_{0}<0.8 \mathrm{~T}\right)$ and by stresses and heating in the ohmic 
solenoid ( $\sim 0.25 \mathrm{~Wb}, \sim 180 \mathrm{~ms})$. The design of ETE incorporates some innovative technological features that resulted in a compact and light weighted device with good plasma accessibility. In the following sections some of the technical characteristics of the main components of the tokamak will be described.

Table 2. Main parameters of the ETE tokamak.

\begin{tabular}{|l|c|c|}
\hline & First phase & Upgrade \\
\hline Major radius $R_{0}$ & $0.30 \mathrm{~m}$ & - \\
Minor radius $a$ & $0.20 \mathrm{~m}$ & - \\
Elongation $\kappa$ & 1.6 & 1.8 \\
Triangularity $\delta$ & $\sim 0.3$ & - \\
Toroidal induction $B_{0}$ & $0.4 \mathrm{~T}$ & $<0.8 \mathrm{~T}$ \\
Plasma current $I_{p}$ & $0.2 \mathrm{MA}$ & $0.4 \mathrm{MA}$ \\
\hline
\end{tabular}

\section{V.1 Vacuum system}

Figure 4 shows a drawing of the vacuum chamber of the ETE tokamak. It is manufactured from Inconel 625 alloy. The relatively high resistivity of Inconel helps to weaken the eddy current effects in the continuous vessel. The thickness of the inner cylindrical wall is $1 \mathrm{~mm}$ and the thickness of the torispherical heads and outer cylindrical wall is $6.35 \mathrm{~mm}$. The internal diameter of the vessel is $0.178 \mathrm{~m}$, the external diameter is $1.219 \mathrm{~m}$ and the height is $1.200 \mathrm{~m}$. The inner cylindrical wall of the vacuum vessel can be removed by grinding and replaced by a smaller diameter tube with the removal of the ohmic heating solenoid. In this way the aspect ratio of ETE can be reduced from 1.5 to 1.3, if necessary in the future.

Standard ConFlat flanges are used so that adequate baking and discharge cleaning up to $200{ }^{\circ} \mathrm{C}$ can been implemented. Short bellows were roll-formed at the extremities of the inner cylindrical wall in order to reduce the thermal stresses during baking. The flanges are distributed around the vacuum chamber in the following configuration: $6 \times 14 \mathrm{CF}$ and $4 \times 250 \mathrm{CF}$ lateral ports, $3 \times 14 \mathrm{CF}$ upward facing ports, $3 \times 14 \mathrm{CF}$ downward facing ports and $42 \times 40 \mathrm{CF}$ small flanges. Several supports welded in the inner wall of the vacuum chamber are used to install rail limiters and diagnostics. The vacuum system consists of a $1500 \mathrm{\ell} / \mathrm{s}$ turbodrag pump backed by a $4 \mathrm{~m}^{3} / \mathrm{h}$ diaphragm pump, plus a $30 \mathrm{~m}^{3} / \mathrm{h}$ rotary pump for maintenance procedures, two piezoelectric valves for gas injection (hydrogen, helium, argon, nitrogen) trough a stainless steel line with independent flow and time control, and a residual gas analyzer (RGA). The vacuum chamber is covered by hot tapes for baking $(16 \mathrm{~kW})$ and a thermal insulation blanket. A base pressure of $8 \times 10^{-8} \mathrm{mbar}$ is easily achieved with baking only up to $110^{\circ} \mathrm{C}$. A butterfly valve placed in front of the turbodrag pump is used to control the gas flow during glow discharge cleaning (maximum $1 \mathrm{kV} / 10 \mathrm{~A}$ in two symmetrical anodes).

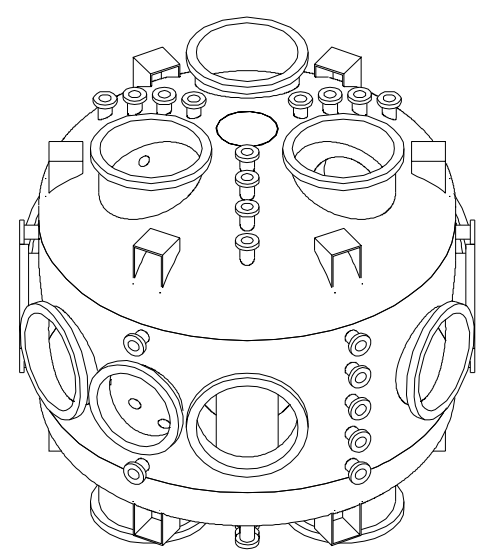

Figure 4. Vacuum chamber of the ETE tokamak.

Electric fields as low as $2 \mathrm{~V} / \mathrm{m}$ are sufficient for breakdown at $2 \sim 3 \times 10^{-4}$ mbar hydrogen pressure with preionization provided by hot filaments $(4 \mathrm{~A} \times 47 \mathrm{~V}, 75 \mathrm{~V}$ polarization). Pre-ionization by electron cyclotron resonance heating will be tried in the future to improve the startup performance of ETE. For this application, a monotron, which is the simplest microwave tube, is under development by the high-power microwave sources group of LAP. The monotron experiment currently under way consists of an electron beam $\left(E_{0}=10 \mathrm{keV}, I_{0}=20 \mathrm{~A}\right)$ that traverses a standing-wave cavity resonator $(f=6.7 \mathrm{GHz}$ in the mode $T M_{020}$ ) producing $30 \mathrm{~kW}$ of output power in the mode $T M_{01}$ (the electronic efficiency is $\eta_{\text {elet }}=0.20$ and the circuit efficiency is $\eta_{\text {circ }}=0.75$ ). Figure 5 shows a particlein-cell code simulation of the electron beam bunching inside the cavity. Presently, the prototype electron gun and resonant cavity are being characterized.

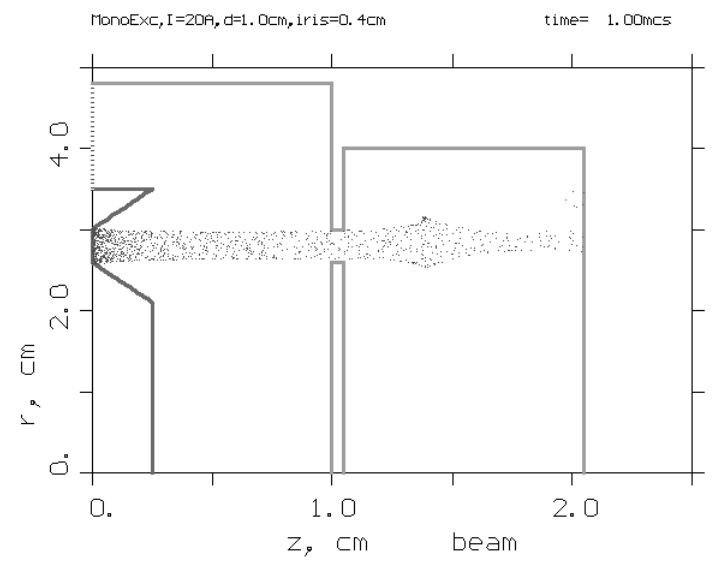

Figure 5. PIC simulation of the monotron showing bunched electron beam.

\section{V.2 Toroidal field coils}

The toroidal field (TF) coil uses a D-shaped minimum stress design shown in Figure 6. Each one of the 12 single turns of the TF coil is free to expand in the poloidal plane. 
The 12 turns are connected in series by a system of current feed rings, illustrated in Figure 7, that compensate the stray magnetic field. The height of the TF coil is $1.68 \mathrm{~m}$ and the diameter is $1.64 \mathrm{~m}$, leading to a magnetic field ripple < $0.3 \%$ at the plasma edge.

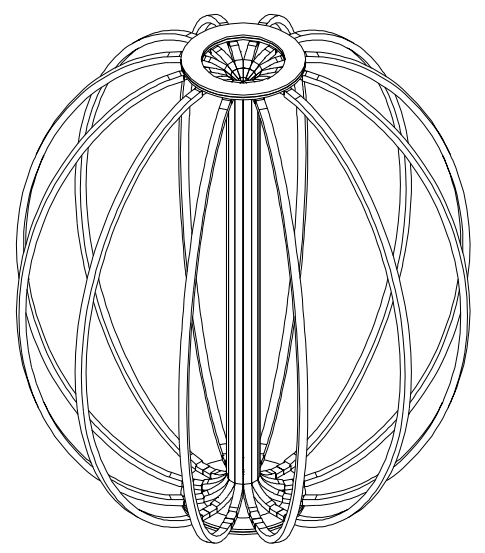

Figure 6. Toroidal field coil of the ETE tokamak.

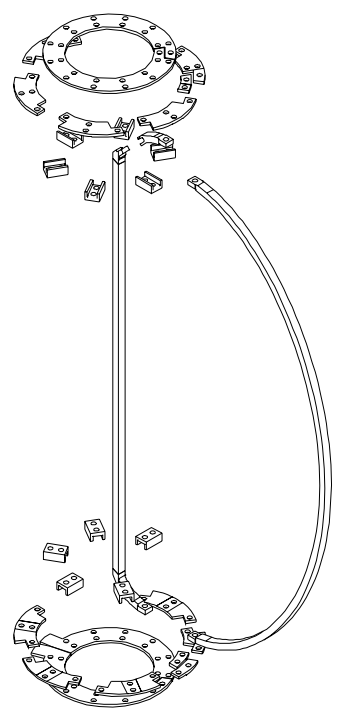

Figure 7. Exploded view of one turn of the TF coil and of the current feed rings.

Electron beam welding was used in all the fixed joints to maintain the half-hard condition of the $7.3 \mathrm{~cm}^{2}$ cross section copper bars, which constitute each turn of the coil. The external legs of the TF coil were bend and machined to the bending moment free shape, and the internal legs were machined to their final trapezoidal cross-section. The center stack, with a diameter of $12.4 \mathrm{~cm}$, is water cooled between shots. Since the current density is constant over the length of the coil, each turn is water cooled in its entire length in order to reduce the stress end effects due to temperature variations. Copper tubes with an internal diameter of $5.75 \mathrm{~mm}$ were pressed into slots machined in the bars of the TF coil and soft-welded or fixed to each bar for water cooling.
The demountable single bolt joints used in the TF coil are illustrated in Figure 8. Prototypes of these joints were submitted to static stress tests to determine the actual stress limit. Figure 9 shows the stress-strain diagram for the external demountable joint. Both the external (superposition) and internal (insertion) joints yielded at a stress level equivalent to $\sim 1 \mathrm{~T}$ magnetic field, as predicted in the design (a $1 \mathrm{~T}$ toroidal magnetic field corresponds to a thin-shell torus constant tension of $\sim 28 \mathrm{kN}$ ). This result indicates that the operation of the TF coil should be limited to a maximum toroidal field of $0.8 \mathrm{~T}$, to keep a safety margin greater than $50 \%$.

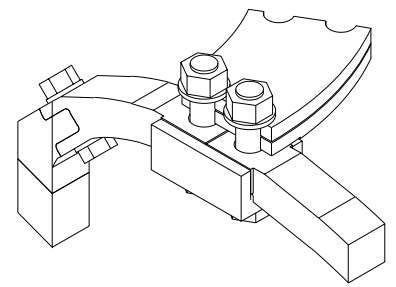

Figure 8. Single bolt demountable joints of the TF coil.

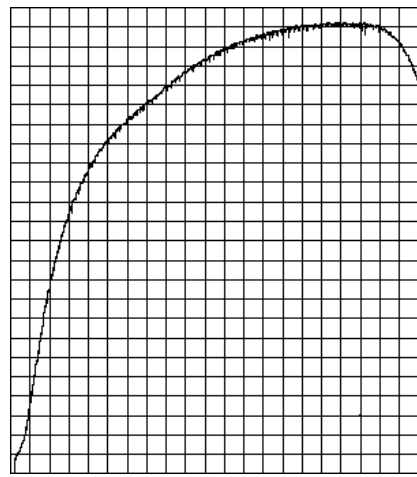

Figure 9. Stress-strain diagram for the external (superposition) demountable joint of the TF coil. Each horizontal division corresponds to a displacement of $1 \mathrm{~mm}$ and each vertical division to a force increment of $3.07 \mathrm{kN}$.

\section{V.3 Poloidal field coils}

In the design of the ETE tokamak a minimal set of coils was adopted for the poloidal field (PF) coils system, as illustrated in Figure 10. It comprises: (1) the plasma magnetizing coils system, formed by the ohmic heating solenoid in series with two pairs of compensation coils, (2) a pair of equilibrium field coils, and (3) a pair of elongation coils. Table 3 summarizes the final geometric parameters of the PF coils, measured during the fabrication procedure. The mean radial dimension indicated for the ohmic heating solenoid corresponds to the equivalent radius for an infinitely long solenoid. Some of the coils allow small adjustments in the vertical position, indicated in the column labelled $Z_{\text {range }}$. The width $(\Delta R, \Delta Z)$ corresponds to the region filled by the conductors. 
Table 3. Geometrical parameters of the poloidal field coils.

\begin{tabular}{|c|c|c|}
\hline \hline \multicolumn{3}{|c|}{ Coil denomination } \\
\hline \hline \multicolumn{3}{|c|}{ Ohmic Heating Solenoid } \\
Internal Compensation Coils \\
External Compensation Coils \\
\hline \multicolumn{3}{|c|}{ Equilibrium Coils } \\
Elongation Coils \\
\hline \hline $\bar{R}[\mathrm{~m}]$ & $\bar{Z}[\mathrm{~m}]$ & $Z_{\text {range }}[\mathrm{m}]$ \\
\hline \hline 0.07246 & 0 & - \\
0.1022 & \pm 0.6606 & - \\
0.6512 & \pm 0.8797 & $0.87 \sim 0.96$ \\
\hline 0.7002 & \pm 0.3900 & $0.32 \sim 0.45$ \\
0.2001 & \pm 0.8300 & $0.79 \sim 0.87$ \\
\hline \hline$\Delta R[\mathrm{~m}]$ & $\Delta Z[\mathrm{~m}]$ & $N R \times N Z$ \\
\hline \hline 0.0192 & 1.3054 & $2 \times 130$ \\
0.021 & 0.100 & $2 \times 10$ \\
0.010 & 0.020 & $1 \times 2$ \\
\hline 0.040 & 0.040 & $4 \times 4$ \\
0.040 & 0.040 & $4 \times 4$ \\
\hline
\end{tabular}

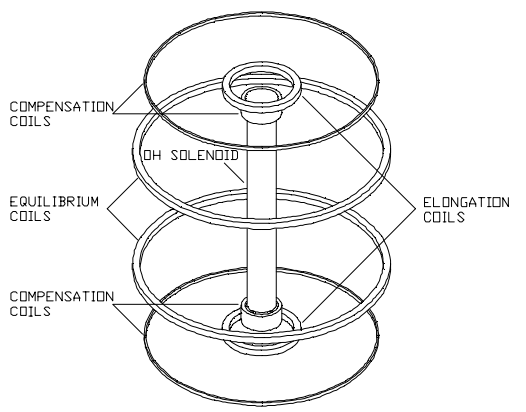

Figure 10. PF coils system of the ETE tokamak.

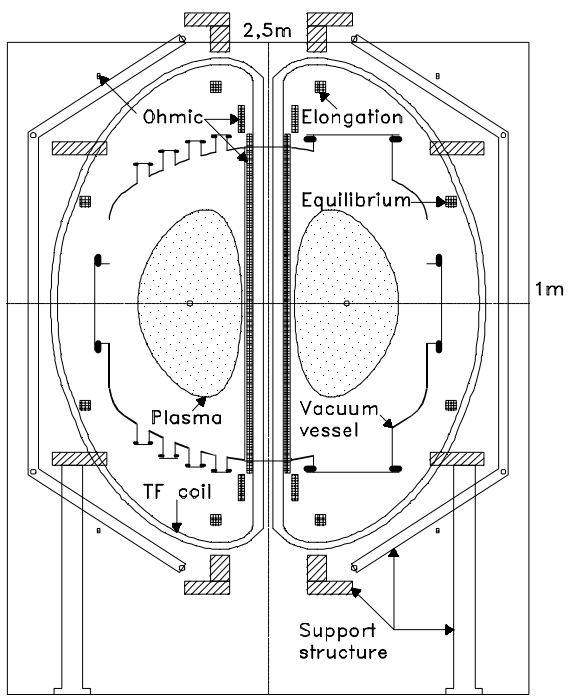

Figure 11. Cross-section of the ETE tokamak.

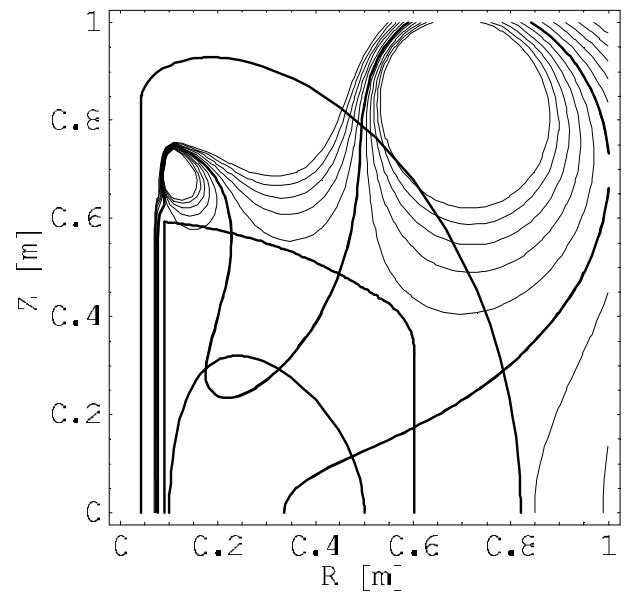

Figure 12. Magnetizing flux contours on the poloidal plane. The heavy contour indicates a poloidal flux of $0.25 \mathrm{~Wb}(2 \times 7.8 \mathrm{MA}$ turns in the $\mathrm{OH}$ solenoid for double-swing operation) with a $2 \%$ increment between contours. The plasma outline, and the vacuum vessel and toroidal field coil centerlines are also displayed.

The position of the PF coils relative to the main components of the tokamak is shown in Figure 11. The location of all the PF coils was carefully studied to allow good diagnostic access. The ohmic heating solenoid $(\mathrm{OH})$ fits the narrow gap between the TF coil and the inner wall of the vacuum vessel. In ETE the $\mathrm{OH}$ solenoid is formed by two layers of a square cross-section $(8.81 \mathrm{~mm} \times 8.81 \mathrm{~mm})$, water cooled hollow conductor ( $4.93 \mathrm{~mm}$ diameter central hole) and was wound around the central column of the TF magnet. The remaining PF coils were wound using a slightly different square cross-section $(9 \mathrm{~mm} \times 9 \mathrm{~mm})$, water cooled hollow conductor ( $5 \mathrm{~mm}$ diameter central hole). All the PF coils are insulated with Kapton, fiberglass tapes and final epoxy resin impregnation. The magnetizing coils system is capable of providing a $0.248 \mathrm{~Wb}$ flux variation with double swing operation within the admissible stress limit for halfhard copper, for a maximum current of $\sim 30 \mathrm{kA}$.

In the initial phase of operation the solenoid will be driven to about half its maximum flux swing capability. In the extended phase the solenoid operation will be limited essentially by the maximum adiabatic temperature rise during each shot. The two pairs of passive compensation coils have their location and number of windings optimized to produce, together with the $\mathrm{OH}$ solenoid, a minimum error field over a large region near the midplane of the plasma (hexapole field cancellation), as shown in Figure 12. These coils must also be water cooled since they are fabricated with basically the same conductor used in the $\mathrm{OH}$ solenoid and are connected in series. The remaining two pairs of PF coils supply the time-varying vertical field necessary for plasma position control and for some limited control of the plasma elongation and dispersion of field lines. The central stack $(\ell=$ $1.87 \mathrm{~m}, d=168.8 \mathrm{~mm}$ ) was the most challenging piece completely manufactured in the workshop of LAP, as well as all the PF coils. 


\section{V.4 Mechanical structures}

Figure 13 shows a drawing of the support structure of the ETE tokamak. It consists basically of two crowns and two rings of insulating material (Tufenol) placed symmetrically with respect to the midplane of the device.

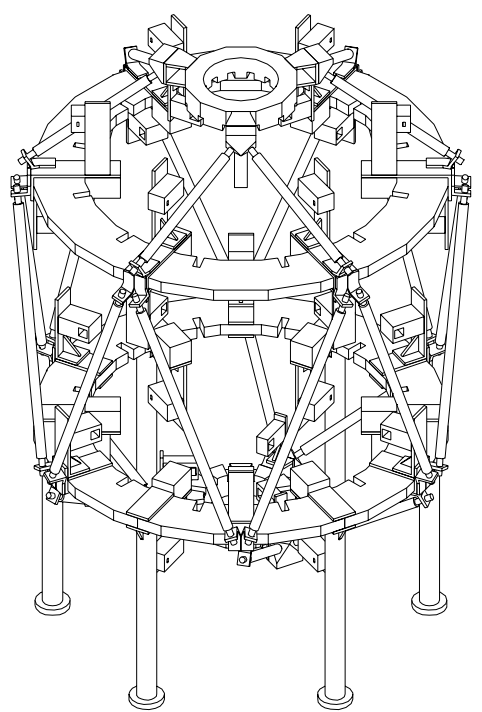

Figure 13. Structure for supporting the vacuum vessel and coils of ETE.

The TF coils are inserted in slots cut in the crowns and rings, which leave the coils free to move in the poloidal plane but resist the out of plane forces. Adjustable stainless steel rods, manufactured from mechanical tubes, connect the crowns and rings, forming a rigid truss structure. The PF coils are attached to the crowns and rings by simple L-shaped supports and clamps, which allow some control of the coils position. The vacuum vessel is placed on plastic shock absorber blocks attached to the support legs and to the upper and lower rings.

The entire assembly is modular and allows very good access for diagnostics. Simple engineering calculations show that the structure is capable to resist the normal and fault electromagnetic loads, but a more careful analysis is necessary to assess the operating limits of the TF coil $\left(B_{0} \leq\right.$ $0.8 \mathrm{~T}$ ) and the structural response of the vacuum vessel to the dynamic forces during a large plasma disruption. The support structure is mounted on a strong aluminum sustentation structure (with toroidal break), which is fixed to a concrete block on the ground level of the tokamak laboratory, giving access to the capacitor banks, power supplies and water cooling equipment. All the mechanical structures have been fabricated in the Institute's workshop, and have been assembled and adjusted in the tokamak hall with an overall precision of $2 \mathrm{~mm}$.

\section{V.5 Power supplies}

The power supplies system for ETE is based entirely on capacitor banks for energy storage. There are four independent banks: toroidal, magnetizing, equilibrium and elongation.
The toroidal capacitor bank has a total energy of $1.13 \mathrm{MJ}$ and is designed to supply a current of $\sim 50 \mathrm{kA}$ to the toroidal field coil during about $100 \mathrm{~ms}$, producing a magnetic field of $\sim 0.4 \mathrm{~T}$ with $\pm 1 \%$ fluctuations. Low voltage electrolytic capacitors, high current thyristors and diodes are used in a two modules configuration: fast bank $(1.25 \mathrm{kV}, 309 \mathrm{~kJ})$ and slow bank $(360 \mathrm{~V}, 821 \mathrm{~kJ}$ fired sequentially in 8 stages $)$. Figure 14 shows the current waveform in the toroidal field coil, obtained by numerical simulation.

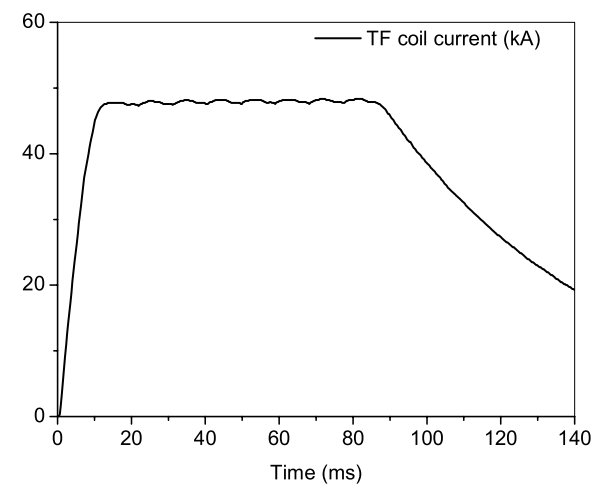

Figure 14. Current waveform in the TF coil, obtained by numerical simulation.

The magnetizing bank or ohmic heating bank has a total energy of $988 \mathrm{~kJ}$ and is designed for double swing operation. This bank uses high voltage oil capacitors, ignitrons and diodes in three modules: charge $(8 \mathrm{kV}, 467 \mathrm{~kJ})$, start-up $(8 \mathrm{kV}, 296 \mathrm{~kJ})$ and flattop $(7.5 \mathrm{kV}, 225 \mathrm{~kJ}$, presently fired in 2 stages). In this configuration the magnetizing bank has the capability of driving and maintaining a plasma current of $\sim 200 \mathrm{kA}$ during $15 \mathrm{~ms}$. Figure 15 shows the current waveforms in the plasma and in the double swing $\mathrm{OH}$ solenoid, obtained by numerical simulation.

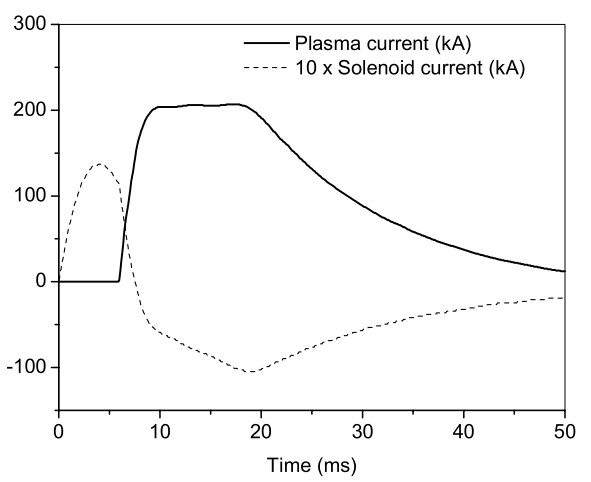

Figure 15. Waveforms of the current in the plasma and 10 times the current in the double swing $\mathrm{OH}$ solenoid, obtained by numerical simulation.

The equilibrium bank has a total energy of $140 \mathrm{~kJ}$ and comprises two modules: fast bank $(7.5 \mathrm{kV}, 90 \mathrm{~kJ})$ and slow 
bank $(360 \mathrm{~V}, 50 \mathrm{~kJ}$ fired in 5 stages), which use an ignitron triggering circuit and thyristor triggering circuits, respectively. The capacitor bank is designed to supply a current of $\sim 6 \mathrm{kA}$ during $25 \mathrm{~ms}$ to the equilibrium field coils. The elongation bank is still in the design phase since its implementation is not essential during the initial operation of the tokamak.

All activities involving the reconditioning and testing of (mostly secondhand) capacitor banks, fabrication of support structures, and development of triggering and safety circuits used in ETE were conducted by the electronics workshop of LAP. Presently, the energy of the capacitor banks is being continuously increased by adding more capacitor modules as well as by rising the voltage rating to its maximum to reach the first operational phase listed in Table 2.

The magnetizing bank is being provisionally operated in low energy single polarity mode while the effects of eddy currents induced on the vacuum vessel are evaluated and compensation for error fields in the start-up phase is introduced. With this low energy configuration the plasma current is limited to the 40 to $60 \mathrm{kA}$ range corresponding to a pulse duration in the 8 to $5 \mathrm{~ms}$ range $(12 \mathrm{~ms}$ with glow discharge cleaning). Figure 16 shows the evolution of the plasma current in various phases during the operation of ETE.

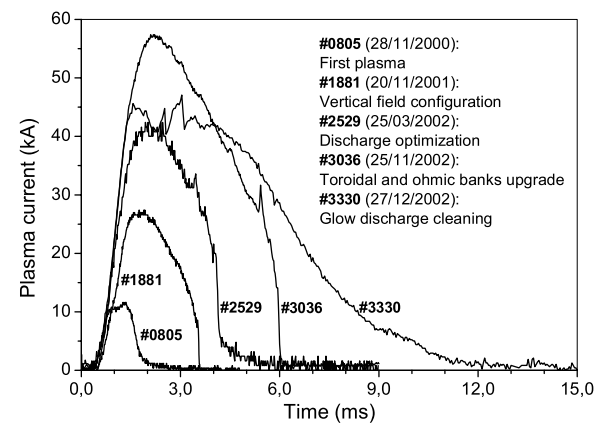

Figure 16. Evolution of the plasma current in various phases during the operation of ETE.

\section{V.6 Control and data acquisition systems}

The ETE tokamak is computer operated with an electropneumatic safety system and complete galvanic insulation. The control is based on CAMAC modules and data acquisition on a VME bus standard. An optical link provides an insulation of $2 \mathrm{kV}$ ( $15 \mathrm{kV}$ in the near future) between the control computer and the CAMAC modules. The galvanic insulation between the CAMAC crate and the hazardous environment is achieved by optical and pneumatic systems. All the components of the control system, including triggering circuits and pneumatic actuators, are functional and the control software is under continuous development using the $\mathrm{C}$ language and HTML interfaces. The VME modules will be introduced progressively with the installation of the diagnostic systems.

\section{V.7 Diagnostic systems}

A complete set of fundamental diagnostics is planned for ETE. Table 4 lists the diagnostics proposed for implementation during the initial (inductive) phase of operation, together with the plasma parameters to be measured.

Table 4. Plasma diagnostics to be installed in ETE.

\begin{tabular}{|c|}
\hline \hline Diagnostic \\
\hline \hline Magnetics \\
$\mathrm{H}_{\alpha}$ detectors, bolometers \\
Hard X-ray monitors \\
Optical spectrometers \\
Thomson scattering \\
Fast CCD camera \\
Electrostatic probes \\
Low-energy lithium beam probe \\
Far infrared laser interferometer \\
Arrays of soft X-ray diodes \\
\hline \hline Plasma Parameters \\
\hline Magnetic reconstruction, magnetic activity \\
Radiated power \\
Fast electrons, magnetic activity \\
Electron temperature and impurities \\
Electron temperature and density profiles \\
Optical imaging \\
Plasma edge parameters \\
Plasma edge parameters \\
Integrated density \\
Electron temperature imaging \\
\hline
\end{tabular}

An initial set of electromagnetic diagnostics is already installed, comprising: three Rogowski coils to measure the currents in the toroidal, equilibrium and ohmic circuits, one Rogowski coil placed inside the vessel to measure the plasma current and a second one outside the vessel to measure the induced eddy current, twelve loop voltage coils in different positions (one placed inside the vessel), four fixed magnetic pickup coils $\left(B_{r}, B_{z}\right)$ protected by the graphite limiter for magnetohydrodynamic and magnetic field measurements, two movable magnetic pickup coils $\left(B_{r}, B_{z}\right.$ and $B_{\phi}$ ) at the midplane, and one movable electrostatic probe also at the midplane. A visible light spectrometer (12 $\AA / \mathrm{mm}$ ) for impurity emission detection and an $H_{\alpha}$ detector with interference filter $(\Delta \lambda=13.52 \mathrm{~nm})$ have been installed. An hard X-ray detector is being used to map the radiation pattern around the machine. One fast CCD camera with speed up to 500 FPS and frame velocity up to $1 / 10,000$ has been used on loan. Figs 17 and 18 show the time profiles of the plasma current, loop voltage, vacuum vessel current, vertical component of the magnetic field at the inboard side of the torus $(R=0.0925 \mathrm{~m}, Z=-0.010 \mathrm{~m}), H_{\alpha}$ light emission, and $C I I I$ impurity radiation emission for a typical discharge in the present low energy operating conditions. 


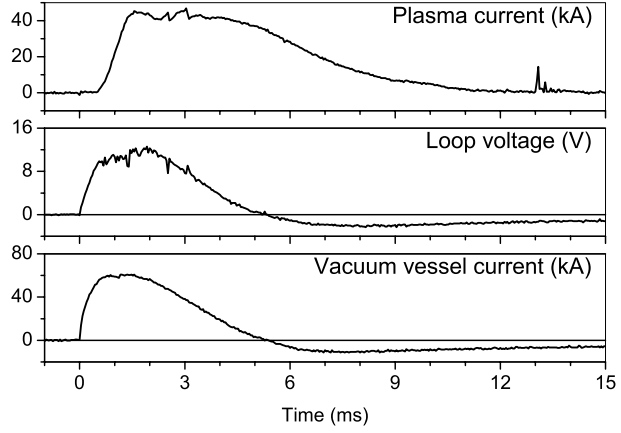

Figure 17. Plasma current, loop voltage and vacuum vessel current for a typical discharge in ETE.

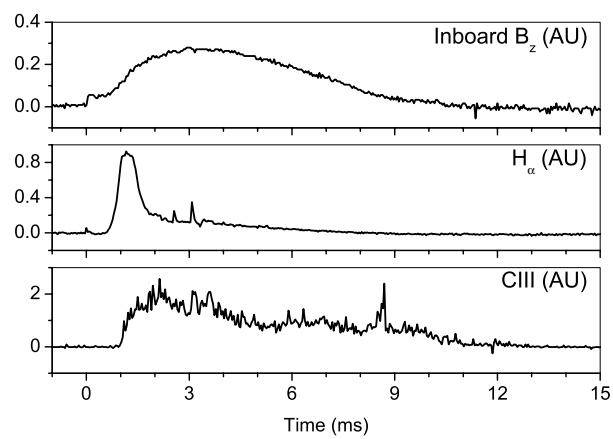

Figure 18. $B_{z}$ component at the inboard side of the torus, $H_{\alpha}$ light and $C I I I$ radiation for a typical discharge in ETE.

The plasma current, loop voltage and $H_{\alpha}$ signals show the occurrence of small internal reconnection events that are typical in the operation of low aspect ratio tokamaks. The current induced in the continuous vacuum vessel is quite high. This is a common feature of most spherical tokamaks, but the eddy current distribution has been measured for the first time in ETE. The measured values compare very well with calculations based on a theoretical model [33]. The problem with eddy currents is less severe in devices like START and MAST that have the poloidal coils mounted inside a large vacuum vessel, but this implies that there is no passive wall stabilization and a very peculiar condition of particle fuelling due to the large volume of the vacuum chamber compared to the plasma volume. In the SUNIST device it is planned to verify the full effect of vessel eddy currents on the plasma discharge with the installation of an elaborate insulating break [16]. Another aspect of the eddy currents is that they affect the magnetic measurements. For example, the loop voltage signal shown in Figure 17 has a large contribution from the current induced in the vessel. Notice that near $t=5 \mathrm{~ms}$ the signal goes through zero, when the induced current also changes its sign and opposes the drop in the plasma current. Presently, methods are being developed to take into account this contribution.

A Thomson scattering system consisting of a $10 \mathrm{~J}, 20 \mathrm{~ns}$ Q-switched ruby laser, a 5-channels polychromator filter with avalanche photodiodes, and collecting lenses capable of scanning 22 different plasma positions along $50 \mathrm{~cm}$ of the laser beam trajectory at the midplane is operational. In the initial tests, plasma temperatures ranging from 20 to $160 \mathrm{eV}$ and densities up to $0.4 \times 10^{20} \mathrm{~m}^{-3}$ have been measured in hydrogen discharges with plasma current of $60 \mathrm{kA}$ amplitude and $5 \mathrm{~ms}$ duration [34]. The highest values obtained for the temperature are consistent with the smallest values of the $H_{\alpha}$ radiation and $C I I I$ impurity. Figs. 19 and 20 show the radial distributions of the electron temperature and the plasma density, respectively, measured $4 \mathrm{~ms}$ after the start of a typical discharge in ETE. The present single channel Thomson scattering system will be gradually upgraded to obtain complete electron temperature and density profiles during each shot. For this purpose, a multipoint operation method using a time-delay technique based on optical fibers of different lengths is being developed.

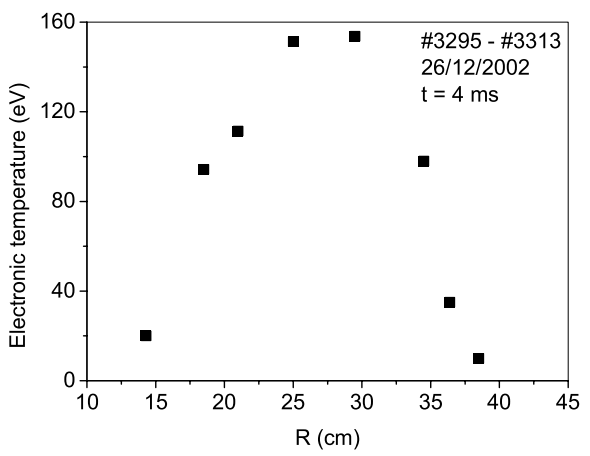

Figure 19. Radial distribution of the electron temperature $4 \mathrm{~ms}$ after the start of the discharge in ETE.

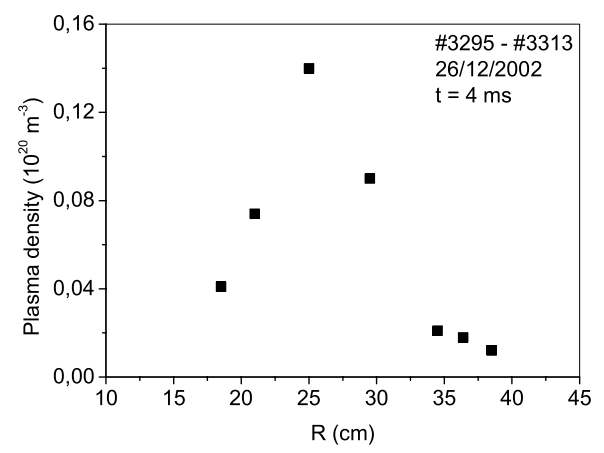

Figure 20. Radial distribution of the plasma density $4 \mathrm{~ms}$ after the start of the discharge in ETE.

A $10 \mathrm{kV}$ fast neutral lithium beam (FNLB) probe with glassy $\beta$-eucryptite source (up to $1 \mathrm{~mA} / \mathrm{cm}^{2}$ ) is presently under development at LAP and will be used for measuring several parameters in the outer region of the plasma. The FNLB, which is currently undergoing final tests, consists of an ion gun (ion source and 3 electrostatic lenses), a sodium based neutralization chamber, a flight line with differential pumping, and a detection system. 
Design of a multipass Michelson interferometer system has been recently completed. This system uses a $50 \mathrm{~W} \mathrm{CO}_{2}$ $\operatorname{laser}\left(\lambda_{\mathrm{CO}_{2}}=10.6 \mu \mathrm{m}\right)$ and a $20 \mathrm{~mW} \mathrm{HeNe}$ laser $\left(\lambda_{\mathrm{HeNe}}=\right.$ $0.6328 \mu \mathrm{m})$. The $\mathrm{CO}_{2}$ laser is injected in the tokamak through a set of flat mirrors and is kept in the plasma by multiple reflections between two spherical mirrors $(R=$ $1.5 \mathrm{~m}$ ). Simulations indicate that it is possible to have 22 passages through the plasma, which correspond to more than two fringes of wavelength shift considering a plasma length of $60 \mathrm{~cm}$ and peak density $0.5 \times 10^{20} \mathrm{~m}^{-3}$. The HeNe laser will be used for alignment purposes and to eliminate errors due to mechanical vibration of the system. An array of soft $\mathrm{X}$-ray diodes, which is in the design phase, will be used to produce images of the temperature in three planes, providing additional information for the magnetic reconstruction procedure, still to be developed.

\section{V.8 Infrastructure}

The main building for ETE was finished in mid 1998. The installations comprise: the tokamak hall $\left(150 \mathrm{~m}^{2}\right.$ containing a 2 ton crane and a $800 \mathrm{~kg}$ lift), the power supplies area $\left(225 \mathrm{~m}^{2}\right.$ for the capacitors banks), the control room $\left(35 \mathrm{~m}^{2}\right)$, the engineering and physics room $\left(30 \mathrm{~m}^{2}\right)$, the computer room $\left(18 \mathrm{~m}^{2}\right)$, the support systems area $\left(50 \mathrm{~m}^{2} \mathrm{re}-\right.$ served for the water cooling system $-10 \mathrm{bar}, 6 \mathrm{~m}^{3} / \mathrm{h}, 6-20^{\circ} \mathrm{C}$ - and the dry compressed air system - 7 bar, $0.5 \mathrm{~m}^{3} / \mathrm{min}$ ), the electrical supply room $\left(50 \mathrm{~m}^{2}\right.$ containing transformers with ultra insulation - $450 \mathrm{kVA}$, plus transformers with standard insulation - $300 \mathrm{kVA}$ ). Finally, the ETE workshop, with $250 \mathrm{~m}^{2}$ in two stories, is used for the fabrication of coils and mechanical parts plus the development of electronic circuits.

\section{Acknowledgments}

The construction of ETE was partially supported by the Studies and Projects Funding Body (FINEP) and the State of São Paulo Research Foundation (FAPESP). One of the authors (G.O.L.) thanks the International Atomic Energy Agency (IAEA) for financial support to ETE related activities through research contract BRA-10528.

\section{References}

[1] Y-K. M. Peng and D.J. Strickler. "Features of spherical torus plasmas”. Nuclear Fusion, 26 (6), 769 (1986).

[2] H. Bruhns, R. Brendel, G. Raupp, and J. Steiger. "Study of the low aspect ratio limit tokamak in the Heidelberg spheromak experiment". Nuclear Fusion, 27 (12), 2178 (1987).

[3] G.A. Collins, G. Durance, G.R. Hogg, J. Tendys, and P.A. Watterson. "Small aspect ratio tokamak configurations generated by rotating magnetic field current drive". Nuclear Fusion, 28 (2), 255 (1988).

[4] P.K. Browning, G. Cunningham, R. Duck, S.J. Gee, K.J. Gibson, D.A. Kitson, R. Martin, and M.G. Rusbridge. "Injection and sustainment of plasma in a preexisting toroidal field using a coaxial helicity source". Physical Review Letters, 68 (11), 1722 (1992)
[5] E. Del Bosco, R.J. Colchin, G. Cunningham, et al. "Results from the START tokamak". Proceedings of the $1^{\circ}$ Congresso Brasileiro de Física dos Plasmas, Vol. I - Invited Papers: 212227. INPE, São José dos Campos, 1991.

[6] A. Sykes, E. Del Bosco, R.J. Colchin, et al. "First results from the START experiment". Nuclear Fusion, 32 (4), 694 (1992).

[7] A. Sykes and the START, NBI, MAST and Theory Teams. "The spherical tokamak programme at Culham". Nuclear Fusion, 39 (9Y), 1271 (1999).

[8] T. Maekawa, H. Tanaka, M. Uchida, et al. "Start-up and formation of ST plasmas by ECH on the LATE device". 19th Fusion Energy Conference, Lyon, France, 2002. Paper EX/P416, IAEA, Vienna, 2002.

[9] R. Kaita, R. Majeski, R. Doerner, et al. "Liquid lithium limiter effects on tokamak plasmas and plasma-liquid surface interactions". 19th Fusion Energy Conference, Lyon, France, 2002. Paper EX/P4-19, IAEA, Vienna, 2002.

[10] Y. Ono, T. Kimura, E.Kawamori, et al. "High-beta characteristics of first and second-stable spherical tokamaks in reconnection heating experiments of TS-3". Nuclear Fusion $\mathbf{4 3}$, 789 (2003).

[11] F. Alladio, A. Mancuso, P. Micozzi, et al. "PROTOSPHERA". Report RT/ERG/FUS/2001/14, ENEA Centro Ricerche Frascati, Rome, Italy, 2001

[12] A.J. Redd, B.A. Nelson, T.R. Jarboe, et al. "Current drive experiments in the helicity injected torus (HIT-II)". Physics of Plasmas, 9 (5), 2006 (2002).

[13] M. Nagata, T. Oguro, Y. Kagei, et al. "Coaxial helicity injection and $\mathrm{n}=1$ relaxation activity in the HIST spherical torus". 19th Fusion Energy Conference, Lyon, France, 2002. Paper EX/P4-17, IAEA, Vienna, 2002.

[14] R.J. Fonck, G. Garstka, S. Diem, et al. "Performance and stability limits at near-unity aspect ratio in the Pegasus toroidal experiment”. 19th Fusion Energy Conference, Lyon, France, 2002. Paper EX/P3-09, IAEA, Vienna, 2002.

[15] A. Ejiri, S. Shiraiwa, Y. Takase, et al. "Ion temperature increase during MHD events on the TST-2 spherical tokamak". 19th Fusion Energy Conference, Lyon, France, 2002. Paper EX/P4-10, IAEA, Vienna, 2002.

[16] Y. He. "Research program of spherical tokamak in China". Proceedings of the Joint Meeting of the 2nd International Atomic Energy Agency Technical Committee Meeting on Spherical Tori and the 7th International Spherical Torus Workshop, INPE, São José dos Campos, SP, Brazil, 2001.

[17] V.K. Gusev, A.S. Ananiev, I.N. Chugunov, et al. "New results from Globus-M spherical tokamak". 19th Fusion Energy Conference, Lyon, France, 2002. Paper EX/P3-10, IAEA, Vienna, 2002.

[18] A. Sykes. "Overview of recent spherical tokamak results". Plasma Physics and Controlled Fusion, 43, A127 (2001).

[19] B. Lloyd for the MAST team. "Overview of recent experimental results on MAST". 19th Fusion Energy Conference, Lyon, France, 2002. Paper OV/2-3, IAEA, Vienna, 2002.

[20] R. Maingi et al. "Recent results from the National Spherical Torus Experiment". Plasma Physics and Controlled Fusion, 45, 657 (2003).

[21] A. Montes, G.O. Ludwig. "Electron Bernstein wave current drive in the start-up phase of a tokamak discharge". Plasma Physics and Controlled Fusion, 28 (12A), 1765 (1986). 
[22] R.M.O. Galvão, L.C.S. Góes, G.O. Ludwig, A. Montes, and M. Ueda. "Conceptual design of a radio frequency driven compact tokamak". In P.H. Sakanaka (ed.), Fusion Energy and Plasma Physics, Proceedings of the First Energy Independence Conference, pp. 471-483. World Scientific, Singapore (ISBN-9971-50-749-8) 1988.

[23] D.C. Robinson. "First Energy Independence Conference: Fusion Energy and Plasma Physics". Nuclear Fusion, 28 (2), 331 (1988)

[24] G.O. Ludwig, A. Montes, and P.H. Sakanaka. "Preliminary design of a small aspect ratio tokamak". Research Using Small Tokamaks, Proceedings of the IAEA Technical Committee Meeting on Research using Small Tokamaks, Nice, France, 1988. IAEA Technical Document 519: 111-123, Vienna, 1989.

[25] G.O. Ludwig, Y. Aso, J.J. Barroso, J.L. Ferreira, R.M.O. Galvão, A. Montes, G.M. Sandonato, M. Ueda, W.P. Sá, A.G. Tuszel, and L.C.S. Góes. "The Proto-ETA small aspect ratio experiment". Improving Tokamak Performance through Innovations from Small Fusion Experiments, Proceedings of the IAEA Technical Committee Meeting on Research using Small Tokamaks, Washington, USA, 1990. IAEA Technical Document 604: 159-174, Vienna, 1991.

[26] G.O. Ludwig and A. Montes. "Plasma performance of low aspect ratio tokamaks - the TBR-E experiment". Proceedings of the $1^{\circ}$ Congresso Brasileiro de Física dos Plasmas, Vol. I, Invited Papers: 332-345. INPE, São José dos Campos, 1991.

[27] G.O. Ludwig. "Anteprojeto de engenharia do tokamak ETE". INPE report 5529-PRE/1796 (in Portuguese), INPE, São José dos Campos, 1993.
[28] G.O. Ludwig. "Theoretical methods in the design of the poloidal field coils for the ETE spherical tokamak", Brazilian Journal of Physics 27 (3), 392 (1997).

[29] G.O. Ludwig, L.F.W. Barbosa, E. Del Bosco, et al. "The Brazilian Spherical Tokamak Experiment". Proceedings of the $3^{\circ}$ Encontro Brasileiro de Física dos Plasmas: 128-131. INPE, São José dos Campos, 1995.

[30] E. Del Bosco, G.O. Ludwig, J.G. Ferreira, et al. "The ETE spherical tokamak: present status of construction". Proceedings of the $4^{\circ}$ Encontro Brasileiro de Física dos Plasmas: 2427. INPE, São José dos Campos, 1996.

[31] E. Del Bosco, G.O. Ludwig, A. Montes, et al. "Status of construction of the ETE spherical tokamak". Proceedings of the $5^{\circ}$ Encontro Brasileiro de Física dos Plasmas: 277-280. SBF, São Paulo, 1998.

[32] G.O. Ludwig, E. Del Bosco, J.G. Ferreira, et al. "First results of the ETE spherical torus". Abstracts of the $6^{\circ}$ Encontro Brasileiro de Física dos Plasmas, Invited Paper: 53 , Campos do Jordão, SP, 2001

[33] G.O. Ludwig. "Calculation of eddy currents in the ETE spherical torus". In I.S. Falconer, R.L. Dewar and J. Khachan (eds.), Proceedings of the Eleventh International Congress on Plasma Physics, AIP Conference Proceedings 669: 573-576 (ISBN 0-7354-0133-0) 2003.

[34] L.A. Berni, E. Del Bosco, J.G. Ferreira, et al. "Overview and initial results of the ETE spherical tokamak". 19th Fusion Energy Conference, Lyon, France, 2002. Paper EX/P4-20, IAEA, Vienna, 2002. 